\title{
Weighted gene coexpression network analysis strategies applied to mouse weight
}

\author{
Tova F. Fuller · Anatole Ghazalpour · Jason E. Aten · Thomas A. Drake • \\ Aldons J. Lusis · Steve Horvath
}

Received: 20 March 2007 / Accepted: 1 June 2007/Published online: 1 August 2007

(C) Springer Science+Business Media, LLC 2007

\begin{abstract}
Systems-oriented genetic approaches that incorporate gene expression and genotype data are valuable in the quest for genetic regulatory loci underlying complex traits. Gene coexpression network analysis lends itself to identification of entire groups of differentially regulated
\end{abstract}

Tova F. Fuller, Anatole Ghazalpour contributed equally to this work.

Electronic supplementary material The online version of this article (doi: 10.1007/s00335-007-9043-3) contains supplementary material, which is available to authorized users.

T. F. Fuller · J. E. Aten · A. J. Lusis · S. Horvath Department of Human Genetics, David Geffen School of Medicine, University of California at Los Angeles, Los Angeles, California, USA

\section{A. Ghazalpour · A. J. Lusis}

Department of Microbiology, Immunology, and Molecular Genetics, University of California at Los Angeles, Los Angeles, California, USA

T. A. Drake

Department of Pathology and Laboratory Medicine, David Geffen School of Medicine, University of California at Los Angeles, Los Angeles, California, USA

\section{A. J. Lusis}

Department of Medicine, David Geffen School of Medicine, and Molecular Biology Institute, University of California at Los Angeles, Los Angeles, California, USA

\section{S. Horvath}

Department of Biostatistics, School of Public Health, University of California at Los Angeles, Los Angeles, California, USA

S. Horvath $(\bowtie)$

UCLA Human Genetics / Biostatistics, 4357A Gonda Center, P.O. Box 957088, Los Angeles, CA 90095-7088, USA

e-mail: shorvath@mednet.ucla.edu genes - a highly relevant endeavor in finding the underpinnings of complex traits that are, by definition, polygenic in nature. Here we describe one such approach based on liver gene expression and genotype data from an $\mathrm{F}_{2}$ mouse intercross utilizing weighted gene coexpression network analysis (WGCNA) of gene expression data to identify physiologically relevant modules. We describe two strategies: singlenetwork analysis and differential network analysis. Singlenetwork analysis reveals the presence of a physiologically interesting module that can be found in two distinct mouse crosses. Module quantitative trait loci (mQTLs) that perturb this module were discovered. In addition, we report a list of genetic drivers for this module. Differential network analysis reveals differences in connectivity and module structure between two networks based on the liver expression data of lean and obese mice. Functional annotation of these genes suggests a biological pathway involving epidermal growth factor (EGF). Our results demonstrate the utility of WGCNA in identifying genetic drivers and in finding genetic pathways represented by gene modules. These examples provide evidence that integration of network properties may well help chart the path across the gene-trait chasm.

\section{Introduction}

While traditional meiotic mapping methods such as linkage analysis and allelic association studies have been fruitful in identifying genetic targets responsible for Mendelian traits, these methods have been less successful in the identification of pathways and genes underlying complex traits. Integration of gene expression, genetic marker, and phenotype data via genetical genomics strategies is increasingly used in complex disease research (Bystrykh et al. 2005; Chen et al. 
2004; Chesler et al. 2005; Hubner et al. 2005; Mahr et al. 2006; Nishimura et al. 2005; Schadt et al. 2003).

Closely related to "genetical genomics" are "systems genetics" approaches that emphasize network methods to describe the relationship between the transcriptome, physiologic traits, and genetic markers (Drake et al. 2006; Kadarmideen et al. 2006; Schadt and Lum 2006). Here we describe a particular incarnation of a systems genetics approach: integrated weighted gene coexpression network analysis (WGCNA) (Zhang and Horvath 2005; Horvath et al. 2006). By focusing on modules rather than on individual gene expressions, WGCNA greatly alleviates the multipletesting problem inherent in microarray data analysis. Instead of relating thousands of genes to the physiologic trait, it focuses on the relationship between a few (here 12) modules and the trait. Because modules may correspond to biological pathways, focusing the analysis on module eigengenes (and equivalently intramodular hub genes) amounts to a biologically motivated data reduction scheme. WGCNA starts from the level of thousands of genes, identifies clinically interesting gene modules, and finally screens for suitable targets by requiring module membership (high intramodular connectivity) and other application-dependent criteria such as gene ontology or associations with clinical trait-related quantitative trait loci. Genetic marker data allow one to identify the chromosomal locations (referred to as module quantitative trait loci, mQTLs) that influence the module expression profiles. Genetic marker data also allow one to prioritize genes inside trait-related modules. In particular, if a genetic marker is known to be associated with the module expressions, using it to screen for gene expressions that correlate with the SNP allows one to identify upstream drivers of the module expressions. The underlying assumption in such an analysis is that functionally related genes and/ or genetic pathways are regulated by common genetic drivers. We have applied this approach to identify mQTLs that control the expression profiles of a body weight-related module in an $\mathrm{F}_{2}$ population of mice (Ghazalpour et al. 2006). Here we extend these findings to another mouse cross. We also demonstrate the utility of WGCNA in relating distinct subgroups of a population via differential network analysis.

\section{Materials and methods}

The weighted gene coexpression network terminology is reviewed in Table 1 and in the Supplementary Material, Appendix A.

\section{Data description}

We illustrate our methods using data from previously studied $\mathrm{F}_{2}$ mouse crosses. The first $\mathrm{F}_{2}$ data set $(\mathrm{B} \times \mathrm{H}$ cross) was obtained from liver tissue of 135 female mice derived from the $\mathrm{F}_{2}$ intercross between inbred strains $\mathrm{C} 3 \mathrm{H} /$ HeJ and C57BL/6J (Ghazalpour et al. 2006; Wang et al. 2006). The second $F_{2}(B \times D)$ intercross data included liver tissue of $113 \mathrm{~F}_{2}$ mice derived from a cross of two standard inbred strains, C57BL/6J and DBA/2J (Ghazalpour et al. 2006; Schadt et al. 2003). Body weight and related physiologic ("clinical") traits were measured in both sets of mice. We note that $\mathrm{B} \times \mathrm{H}$ and $\mathrm{B} \times \mathrm{D}$ mice differ in some respects. $\mathrm{B} \times \mathrm{H}$ mice are ApoE null (ApoE $-/-)$ and thus hyperlipidemic, whereas $\mathrm{B} \times \mathrm{D}$ mice are wild type $(\mathrm{ApoE}+/+)$. $\mathrm{B} \times \mathrm{H}$ mice were fed a high-fat diet and $\mathrm{B} \times \mathrm{D}$ mice were fed a high-fat, high-cholesterol atherogenic diet. Also, $\mathrm{B} \times \mathrm{H}$ mice were sacrificed at an earlier age (24 weeks) than were the $\mathrm{B} \times \mathrm{D}$ mice $(16$ months).

\section{Coexpression network analysis strategies}

In the following, we present two distinct network analysis approaches: single-network analysis and differential network analysis. The two approaches answer different questions. The single-network analysis defines modules that can then be tested for validity with other data sets. Single-network analysis aims at identifying (a) pathways (modules) and (b) their key drivers (e.g., hub genes) that are present in a given data set. For example, we use all mice of a given $F_{2}$ intercross to identify trait-related modules and mQTLs.

The second strategy, differential network analysis, aims to uncover differences in the modules and connectivity between different data sets (e.g., males versus females). Here we use body weight to arrive at two distinct data sets: lean and obese mice. Each data set is then used to construct a network. Next, the networks are contrasted to find (1) nonpreserved modules, (2) differentially expressed genes, and (3) differentially connected genes. Traditionally, a main goal of studying gene expression data is to relate differences in gene expression profiles to phenotypic differences across different conditions (e.g., different groups of mice). Viewing individual genes in isolation and analysis of differential expression is a wellestablished technique that has already yielded many important insights. On the other hand, differential analysis of network quantities (i.e., quantities describing the relationships between the genes such as intramodular connectivity) is neither as developed nor as widely used, although it has already led to some interesting results. For example, differential analysis of intramodular connectivity was used to identify key differences in expression networks of human and chimpanzee brains (Oldham et al. 2006). 
Table 1 Short glossary of network concepts

\begin{tabular}{|c|c|}
\hline Term & Definition \\
\hline Coexpression network & $\begin{array}{l}\text { We define coexpression networks as undirected, weighted gene networks. The nodes of such a network } \\
\text { correspond to gene expressions, and edges between genes are determined by the pairwise Pearson } \\
\text { correlations between gene expressions. By raising the absolute value of the Pearson correlation to a } \\
\text { power } \beta \geq 1 \text { (soft thresholding), the weighted gene coexpression network construction emphasizes large } \\
\text { correlations at the expense of low correlations. Specifically, } a_{i j}=\left.\operatorname{lcor}\left(x_{i}, x_{j}\right)\right|^{\beta} \text { represents the adjacency. }\end{array}$ \\
\hline Module & $\begin{array}{l}\text { Modules are clusters of highly interconnected genes. In coexpression networks, modules correspond to } \\
\text { clusters of highly correlated gene expressions. }\end{array}$ \\
\hline Connectivity & $\begin{array}{l}\text { For each gene, the connectivity (also known as degree) is defined as the sum of connection strengths with } \\
\text { the other network genes: } k_{i}=\sum_{u \neq i} a_{i u} \text {. In coexpression networks, the connectivity measures how correlated } \\
\text { a gene is with all other network genes. }\end{array}$ \\
\hline $\begin{array}{l}\text { Intramodular connectivity } \\
\quad(\mathrm{kIN})\end{array}$ & $\begin{array}{l}\text { Intramodular connectivity measures how connected, or coexpressed, a given gene is with respect to the } \\
\text { genes of a particular module. The intramodular connectivity may be interpreted as a measure of module } \\
\text { membership. }\end{array}$ \\
\hline Module eigengene & $\begin{array}{l}\text { The module eigengene corresponds to the first principal component of a given module. It can be considered } \\
\text { the most representative gene expression in a module. }\end{array}$ \\
\hline $\begin{array}{l}\text { Module eigengene-based } \\
\text { connectivity (kME) }\end{array}$ & $\begin{array}{l}\text { The module eigengene-based intramodular connectivity measure kME roughly approximates the standard } \\
\text { intramodular connectivity kIN. This measure is determined by correlating the expression profile of a gene } i \\
\text { with the module eigengene of its resident module: } \operatorname{kME}_{i}=\left|\operatorname{cor}\left(x_{i}, \mathrm{ME}\right)\right| \mathrm{l}\end{array}$ \\
\hline Hub gene & $\begin{array}{l}\text { This loosely defined term is used as an abbreviation of "highly connected gene." By definition, genes inside } \\
\text { coexpression modules tend to have high network connectivity. }\end{array}$ \\
\hline Gene significance & $\begin{array}{l}\text { Abstractly speaking, the higher this value, the more significant a gene is. In our application, the gene } \\
\text { significance measures how correlated a gene expression is with a clinical trait. Mouse body weight can be } \\
\text { used to define a physiologic trait-based gene significance measure. Similarly, SNPs can be used to define } \\
\text { SNP-based gene significance measures. }\end{array}$ \\
\hline Module significance & $\begin{array}{l}\text { Module significance is determined as the average of gene significance measures for all genes in a given } \\
\text { module. This measure is highly related to the correlation between module eigengene and the trait. }\end{array}$ \\
\hline mQTLs & $\begin{array}{l}\text { Module quantitative trait loci are chromosomal locations (e.g., SNP markers) that correlate with the module } \\
\text { expression profiles. mQTLs can be defined as hotspots of the expression quantitative trait loci that are } \\
\text { associated with a particular module. }\end{array}$ \\
\hline
\end{tabular}

Single weighted gene coexpression network analysis

In the case of single-network analysis, one uses a single network for modeling the relationship between transcriptome, clinical traits, and genetic marker data. In the following, we describe a typical single-network analysis for finding body weight-related modules and genes. While a single network is the focus, it does not imply that only a single data set is used. Instead, appropriately similar multiple data sets can be used to validate the robustness of module definition and connectivity.

In the following, we provide an overview of singlenetwork analysis strategy, which is depicted in Fig. 1: (1) A weighted gene coexpression network is constructed from genome-wide transcription data. (2) Modules are identified and module centrality measures (intramodular connectivity) are calculated. (3) Network modules are analyzed for biological significance. (4) Genetic loci driving functionally relevant modules within the network are identified. (5) Trait-related mQTLs are used to prioritize genes within physiologically significant modules.
Differential weighted gene coexpression network analysis

We describe another application of WGCNA, differential network analysis, which may be useful in identifying gene pathways distinguishing phenotypically distinct groups of samples. In our example, we identified the 30 mice at both extremes of the weight spectrum in the $\mathrm{B} \times \mathrm{H}$ data and constructed the first network using the 30 leanest mice and the second network using the 30 heaviest mice. For the $i$ th gene, we denote by $k_{1}(i)$ and $k_{2}(i)$ the whole-network connectivity in networks 1 and 2 , respectively. To facilitate the comparison between the connectivity measures of each network, we divide each gene connectivity by the maximum network connectivity, i.e.,

$K_{1}(i)=\frac{k_{1}(i)}{\max \left(k_{1}\right)} \quad$ and $\quad K_{2}(i)=\frac{k_{2}(i)}{\max \left(k_{2}\right)}$.

Next we define a measure of differential connectivity as $\operatorname{DiffK}(i)=K_{1}(i)-K_{2}(i)$, but other measures of differential connectivity could also be considered. 


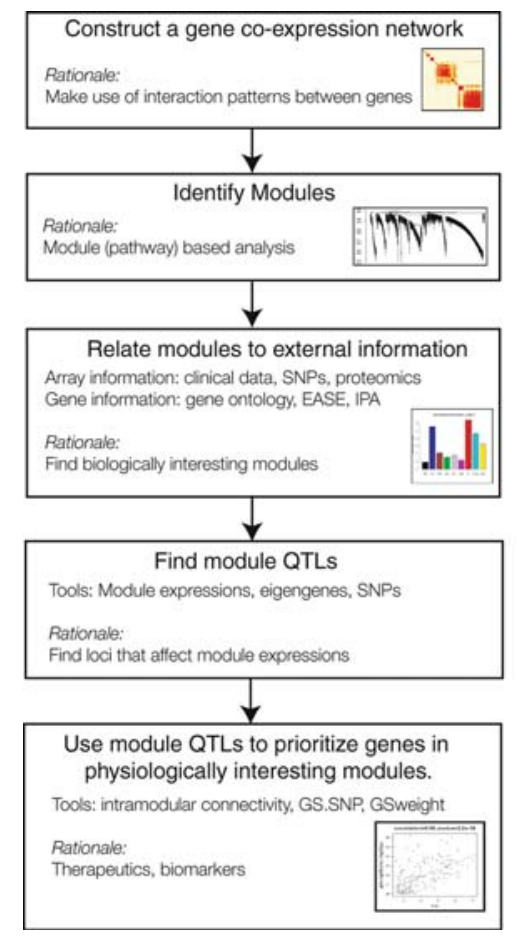

Fig. 1 Overview of weighted gene coexpression network analysis (single-network analysis)

Software availability

R software tutorials and the data for WGCNA can be found at http://www.genetics.ucla.edu/labs/horvath/Coexpression Network/DifferentialNetworkAnalysis/.

\section{Results}

Single network analysis results

A single weighted gene coexpression network was constructed using expression data from livers of 135 female mice of the $\mathrm{B} \times \mathrm{H}$ cross, utilizing the 3421 most connected and varying transcripts from the approximately 23,000 transcripts present on the arrays (Ghazalpour et al. 2006). Using hierarchical clustering, we obtained 12 modules (each designated by a color). Gray denotes genes outside of modules. In this network, the Blue module had the highest module significance score for the physiologic trait of mouse weight $(\mathrm{g})$ (module significance $=0.395$, $p=7.7 \times 10^{-5}$ ), and was also highly significant for abdominal fat pad mass $(\mathrm{g})$ (module significance $=0.323$, $p=0.009$ ). These $p$ values remain significant after Bonferroni correction adjusting for 12 modules. We mention that total mass $(\mathrm{g})$ of other fat depots is also significant (module significance $=0.309, p=0.02$ ), but does not remain significant after Bonferroni correction.
To study the preservation of modules across different $\mathrm{F}_{2}$ intercrosses, we used the $\mathrm{B} \times \mathrm{H}$ module color assignment to cluster the corresponding network in the $\mathrm{B} \times \mathrm{D}$ mouse cross data set (Fig. 2a). A weighted gene coexpression network analysis was constructed using 1953 genes in the $\mathrm{B} \times \mathrm{D}$ data set that have corresponding probes in the $\mathrm{B} \times$ $\mathrm{H}$ data set. We observe that several modules (Red, Blue, Green-yellow, Turquoise, and Green modules being notable examples) are roughly preserved between these two data sets. Figure $2 \mathrm{~b}$ shows a multidimensional scaling (MDS) plot of the $\mathrm{B} \times \mathrm{D}$ data colored by $\mathrm{B} \times \mathrm{H}$ modules. This plot visualizes the pairwise gene dissimilarities by projecting them into a 3-dimensional Euclidean space.

If, in fact, intramodular connectivity (centrality and membership to the Blue module) reflects physiologic significance, one would expect to see a high correlation between kME and GSweight for the Blue module genes. As in Ghazalpour et al. (2006), we find a high correlation between kME and GSweight in the $\mathrm{B} \times \mathrm{H}$ cross $(r=0.47$, $p \leq 10^{-20}$, Fig. 3c). Here we validate this relationship in the $\mathrm{B} \times \mathrm{D}$ cross $\left(r=0.57, p \leq 10^{-20}\right.$, Fig. $\left.3 \mathrm{~d}\right)$.

Figure 3a shows that intramodular connectivity (kME) with regard to the Blue module is preserved between the $\mathrm{B} \times \mathrm{H}$ and the $\mathrm{B} \times \mathrm{D}$ crosses (correlation $r=0.45$, $p \leq 10^{-20}$ ). GSweight was conserved with a Spearman correlation of $0.19\left(p=1.0 \times 10^{-17}\right.$, see Fig. 3b). Network-based gene screening uses both GSweight and kME to find weight-related genes. Note that kME is better preserved than GSweight, which suggests that kME may be a more robust gene-screening variable (see Fig. 3).

A module QTL on chromosome 19

We had previously identified a single nucleotide polymorphism (SNP) marker on chromosome 19 (SNP19) that affected weight and module expression. Table 2 demonstrates the preservation of correlations between the Blue module eigengene MEblue, weight, and SNP19 in both the $\mathrm{B} \times \mathrm{H}$ and the $\mathrm{B} \times \mathrm{D}$ data sets. A relationship was seen between MEblue and weight in both the $\mathrm{B} \times \mathrm{H}$ data $\left(r=0.62, \quad p=1.3 \times 10^{-15}\right)$ and in the $\mathrm{B} \times \mathrm{D}$ cross $\left(r=0.34, p=2.1 \times 10^{-4}\right)$. We note here that while the $p$ values are not adjusted for multiple comparisons, using the most conservative correction-the Bonferroni correction, wherein we multiply the $p$ significance level by the number of modules-still results in a significant correlation between MEblue and weight in the $\mathrm{B} \times \mathrm{H}$ data. More explicitly, in correcting the $p$ value, multiplying $p=1.3 \times 10^{-15}$ by the number of modules (12) leads to a still significant $p=1.6 \times 10^{-14}$. This illustrates the value of using WGCNA to reduce the number of multiple comparisons common to microarray analysis. We note 

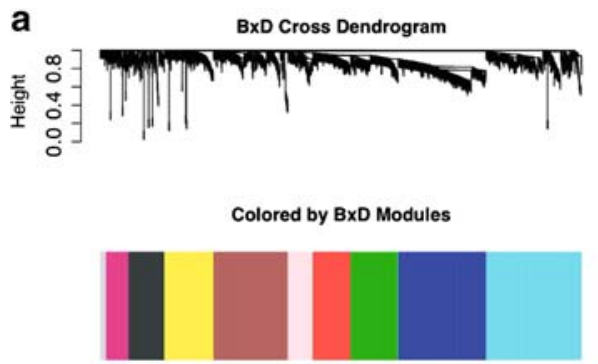

Colored by BxH Modules

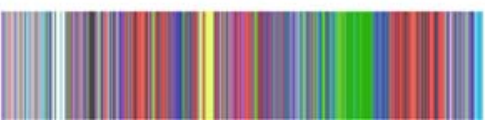

Fig. 2 a (Top) Average linkage hierarchical clustering dendrogram of the $\mathrm{B} \times \mathrm{D}$ cross. (Middle) Visualization of the modules in the $\mathrm{B} \times \mathrm{D}$ network; module colors correspond to branches of the dendrogram shown above. (Bottom) Visualization of rough module

that the mQTL on chromosome 19 had a single-point LOD score of 3.36. While a relatively weaker correlation between SNP19 (d19mit7l) and weight is seen in B $\times \mathrm{D}$ data compared with $\mathrm{B} \times \mathrm{H}$, homozygous animals for the B6 allele of a different marker on chromosome 19 (d19mit63) have significantly different weight from DBA homozygotes (in the $\mathrm{B} \times \mathrm{D}$ cross). This result is

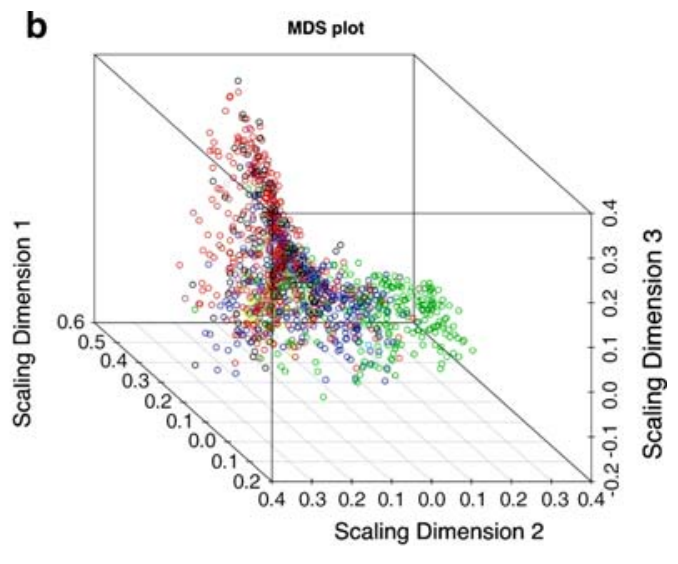

preservation. Here we color the genes by the colors of the original $\mathrm{B} \times \mathrm{H}($ not $\mathrm{B} \times \mathrm{D})$ cross. The fact that colors stay together suggests module preservation. b Multidimensional scaling (MDS) plot of $\mathrm{B} \times \mathrm{D}$ mouse cross data, with coloring by $\mathrm{B} \times \mathrm{H}$ module definitions

consistent with the previous finding that B6 and DBA homozygotes have significantly different subcutaneous fat pad mass (a weight-related trait) (Ghazalpour et al. 2005). It is also possible that the differences in experimental design such as diet, age of the animals, and the status of the Apoe gene could account for the weaker correlation observed in the $\mathrm{B} \times \mathrm{D}$ network.
Fig. 3 a Scatterplot of kME in both crosses. kME describes each eigengene's connectivity to the Blue module. The value for the $\mathrm{B} \times \mathrm{D}$ cross ( $y$ axis) is plotted against the value in the $\mathrm{B} \times \mathrm{H}$ data set ( $x$ axis). $\mathbf{b}$ Scatterplot between GSweight for all genes in the $\mathrm{B} \times \mathrm{D}$ cross ( $y$ axis) and in the $\mathrm{B} \times \mathrm{H}$ cross ( $x$ axis). Colors depict $\mathrm{B} \times \mathrm{H}$ module membership. c Scatterplot between GSweight ( $y$ axis) and $\mathrm{kME}$ ( $x$ axis) in the $\mathrm{B} \times \mathrm{H}$ data set in genes that overlapped with the $\mathrm{B} \times \mathrm{D}$ cross. d Same as (c), except in the $\mathrm{B} \times \mathrm{D}$ cross. Spearman correlation coefficients are reported above all plots
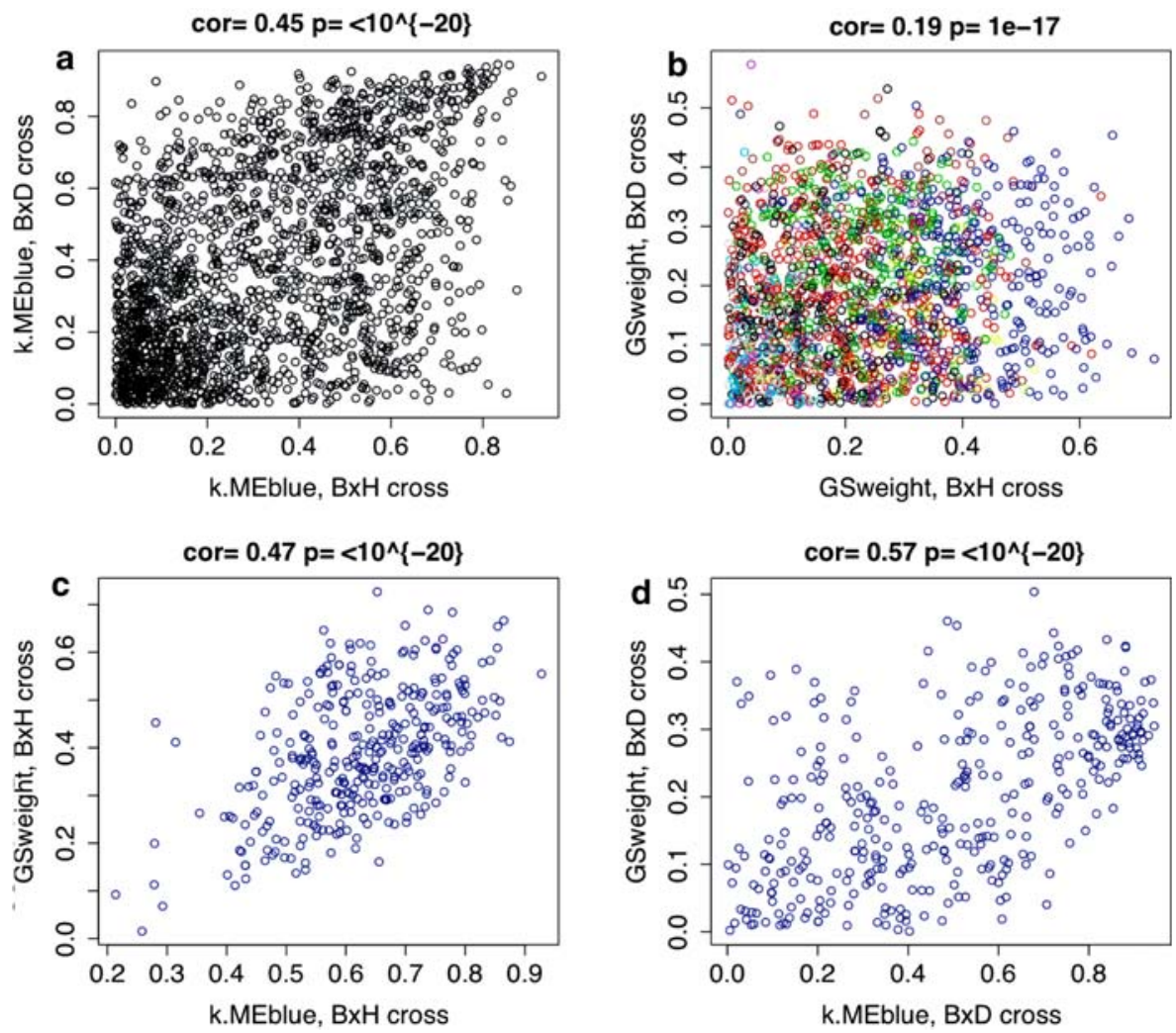
Table 2 Studying the preservation of correlations between the $\mathrm{B} \times \mathrm{H}$ and the $\mathrm{B} \times \mathrm{D}$ mouse cross data

\begin{tabular}{lllll}
\hline Relationship & $\mathrm{B} \times \mathrm{H}$ & & $\mathrm{B} \times \mathrm{D}$ \\
\cline { 2 - 4 } & $r$ & $p$ & $r$ & 0.34 \\
\hline cor(MEblue, weight) & 0.62 & $1.3 \times 10^{-15}$ & 0.25 & 0.0087 \\
cor(MEblue, SNP19) & 0.19 & 0.024 & 0.16 & 0.10 \\
cor(weight, SNP19) & 0.32 & $2.1 \times 10^{-4}$ & 0.57 & $<2.2 \times 10^{-16}$ \\
cor(kME, GSweight) & 0.51 & $<2.2 \times 10^{-16}$ & & 0 \\
\hline
\end{tabular}

MEblue denotes the module eigengene of the Blue module, and weight denotes the mouse body weight. SNP19 denotes the SNP marker (quantitative trait locus) on chromosome 19 that was significantly correlated with body weight in the $\mathrm{B} \times \mathrm{H}$ cross. While the relationship between SNP19 and MEblue can be reproduced in the B $\times$ D data, there is only a weak correlation between SNP19 and weight in the B $\times$ D cross. The highly preserved and strong correlation between GSweight and kME in both crosses shows that highly connected hub genes inside the Blue module are correlated with weight

Using a body weight-related mQTL to prioritize genes inside the Blue module

A SNP marker allows one to define a gene significance measure, GS.SNP, which can be used to prioritize genes within a module.

For the $i$ th gene, GS.SNP $(i)$ is defined as the absolute value of the correlation between the $i$ th gene's expressions and a given SNP's additive marker coding value:

$\operatorname{GS} . \operatorname{SNP}(i)=\mid \operatorname{cor}(x(i), \operatorname{SNP} \mid$.

Additive marker coding reflects the dosage of a given allele; alternatively, one could use dominant or recessive marker coding (see Supplementary Material, Supplementary Table 2).

Observed GS.SNP values are reported in Supplementary Fig. 2a for our simulated module example. We explore the relationship between the GS.SNP values obtained by different marker coding methods in Supplementary Material, Appendix B, and depict the strong relationship between GS.SNP and the traditional LOD score in Supplementary Fig. 3. In short, this figure demonstrates that regardless of whether additive, dominant, or recessive marker coding is used, GS.SNP is highly related to the LOD score values.

Systems genetics gene-screening criteria

As described above, we found a SNP marker on chromosome 19 that is highly related to body weight and to the Blue module expressions. To determine which gene expressions mediate between this mQTL and body weight, it is natural to rank gene expressions based on their correlations with SNP19 and the clinical trait. This suggests to screen for genes with high GS.SNP19 and high GSweight. Furthermore, since the Blue module was found to be related to body weight, it is natural to rank genes by membership to the Blue module, i.e., by intramodular connectivity. Our gene-screening criteria for finding the genetic drivers of body weight are as follows: (1) high association with the body weight, i.e., high values of GSweight; (2) membership and hub status in a trait-related module, i.e., a high value of kME; and (3) high association with a body weight-related mQTL, i.e., high values of GS.SNP. Specifically, we used the 85th percentile of each screening variable, which resulted in nine genes inside the Blue module (Table 3 ). The gene list is quite robust with respect to the percentile as the reader may explore using our online $\mathrm{R}$ software tutorial. An examination of their potential relationship to body weight using the Mouse Genomics Informatics gene ontology database (http://www.informatics.jax.org/) (Eppig et al. 2005) and existing literature yields the following: Fsp27 encodes a pro-apoptotic protein. Nordstrom et al. (2005) found that Fsp27-null mice are resistant to obesity and diabetes. In addition, Fsp27 expression is halved in obese humans after weight loss, and other recent research suggests that Fsp27 regulates lipolysis in white human adipocytes (Nordstrom et al. 2005). A number of the other genes are related to basic biological processes that may be altered in the obese state, which is associated clinically with both the metabolic syndrome and vascular disease, among other conditions. Gpld1 (glycosylphosphatidylinositol-specific phospholipase D1) expression in liver is increased with a high-fat diet in mice, and overexpression is associated with an increase in fasting and postprandial plasma triglycerides and a reduction in triglyceride-rich lipoprotein catabolism (Raikwar et al. 2006). Gene products of $F 7$ and $K n g 2$ are elements of the hemostatic system and may play roles in thrombosis and vascular disease (Kaschina et al. 2004; Reiner et al. 2007; Viles-Gonzalez et al. 2006). Our network-based gene screening method appears to identify biologically relevant genes, considering the evidence from primary literature supporting involvement of these genes in obesity (Fsp27) and/or known obesity-related disorders 
Table 3 Gene-screening results of the single-network analysis

\begin{tabular}{|c|c|c|c|c|c|c|}
\hline \multirow[t]{2}{*}{ Gene } & \multirow[t]{2}{*}{$\mathrm{Chr}$} & \multirow[t]{2}{*}{ ID } & \multicolumn{2}{|c|}{ GS.SNP19 } & \multirow{2}{*}{$\begin{array}{l}\text { GSweight }^{\mathrm{a}} \\
r\end{array}$} & \multirow{2}{*}{$\begin{array}{l}\mathrm{kME}^{\mathrm{b}} \\
R\end{array}$} \\
\hline & & & $r$ & $p$ & & \\
\hline$F 7$ & 8 & МMT00078851 & 0.26 & 0.0021 & 0.67 & 0.85 \\
\hline Kng2 & 16 & МMT00065159 & 0.24 & 0.0048 & 0.66 & 0.81 \\
\hline Pdir & 16 & ММТ00008463 & 0.25 & 0.0032 & 0.62 & 0.80 \\
\hline Slc $30 a 2$ & 4 & МMT00071411 & 0.25 & 0.0032 & 0.58 & 0.79 \\
\hline Lrrc39 & 3 & МMT00078732 & 0.25 & 0.0032 & 0.68 & 0.78 \\
\hline Angl & 14 & МMT00064235 & 0.29 & $5.5 \times 10^{-4}$ & 0.61 & 0.80 \\
\hline Fsp27 & 6 & ММТ00039459 & 0.31 & $2.0 \times 10^{-4}$ & 0.61 & 0.75 \\
\hline Gpld1 & 13 & ММТ00016835 & 0.27 & 0.0014 & 0.54 & 0.77 \\
\hline Sh3d4 & 14 & ММТ00013759 & 0.24 & 0.0048 & 0.60 & 0.79 \\
\hline
\end{tabular}

GS.SNP19 = absolute correlation of gene expressions with SNP19 values; GSweight = absolute correlation of gene expressions with weight $\mathrm{kME}=$ Intramodular connectivity values for nine high-priority genes in the Blue module are shown. Gene symbols, chromosome number, and probe IDs are also shown for each gene

a All $p$ values for GSweight were less than $10^{-12}$

b All $p$ values for GSweight were less than $10^{-12}$

(diabetes, metabolic syndrome, and vascular disease). Other genes identified by this method may be novel candidates. As such, these results should be considered a starting point for subsequent experimentation to explore involvement of these genes in obesity.

Sector plots for identifying differentially expressed and differentially connected genes

Differential network analysis is concerned with identifying both differentially connected and differentially expressed genes. To measure differential gene expression between the lean and the obese mice, we use the absolute value of the Student $t$-test statistic. Plotting $\operatorname{Diff} K$, the difference in connectivity between lean and obese mice, versus the $t$-test statistic value for each gene gives a visual demonstration of how difference in connectivity relates to a more traditional $t$-statistic describing difference in expression between the two networks.

Figure 4a shows a scatterplot of Diff $K$ vs. the $t$ statistic. Eight sectors of the plot with high absolute values of Diff $K$ $(>0.4)$ and/or $t$-statistic values $(>1.96)$ are shown. Horizontal lines depict sector boundaries based on $t$-statistic values, and vertical lines depict boundaries based on Diff $K$. These eight sectors are marked by numbers in Fig. 4a. To assign a significance level ( $p$ value) to a gene's Diff $K$ value or to its membership in a particular sector defined by Diff $K$ and $t$ statistic, we use a permutation test approach that randomly permutes the microarray sample labels. The permutation test contrasts networks built by randomly partitioning the 60 mice into two groups. We consider the number of genes inside a given sector (which is defined by thresholding the $t$ statistic and Diff $K$ as described above) in determining significance level. Figure $4 \mathrm{~b}$ demonstrates the same information except network membership is permuted. Based on 1000 random permutations, sector membership was found to be significant for sectors 2, 3, and 6 with $p \leq 1.0 \times 10^{-3}$. Membership in sector 5 was significant with $p \leq 1.0 \times 10^{-2}$.

Functional enrichment analysis of sector 3 genes

We analyzed 61 sector 3 genes that were both highly connected in network 1 and lowly connected in network 2 for functional enrichment using the DAVID database (Dennis et al. 2003). This software, which is free and available for download at http://www.d.abcc.ncifcrf.gov/home.jsp, calculates the $p$ value for the extent of enrichment of a given biological pathway/set by performing Fisher's exact test. We focused on sector 3 for two reasons. First, sector 3 members had extreme values of $\operatorname{Diff} K$ as well as high $t$ statistic values. Also, as one can readily see from Fig. 4a, a high proportion of Yellow module genes were found in this module, based on network 1 module definitions. These Yellow module genes were lowly connected in network 2, and therefore were annotated as Gray module (background) members in a module assignment scheme based on network 2. This result suggests that in a pathophysiologic state (mouse obesity), the Yellow module can no longer be found.

Results for this analysis that were significant at $p<0.05$ level are shown in Table 4 . These genes were markedly 
Fig. 4 Sector plots of differential network analysis. In (a) and (b), difference in connectivity (Diff $K)$ is plotted on the $x$ axis, and $t$-test statistic values are plotted on the $y$ axis. Horizontal lines indicate a difference in connectivity of -0.4 and 0.4 , whereas vertical lines depict a $t$-statistic value of -1.96 or 1.96 . a Observed Diff $K$ and $t$-statistic values: Genes are colored based on network 1 module definitions. Numbers indicate sectors $1-8$. b Corresponding sector plot for a permuted network where array samples in data sets 1 and 2 were randomly permuted
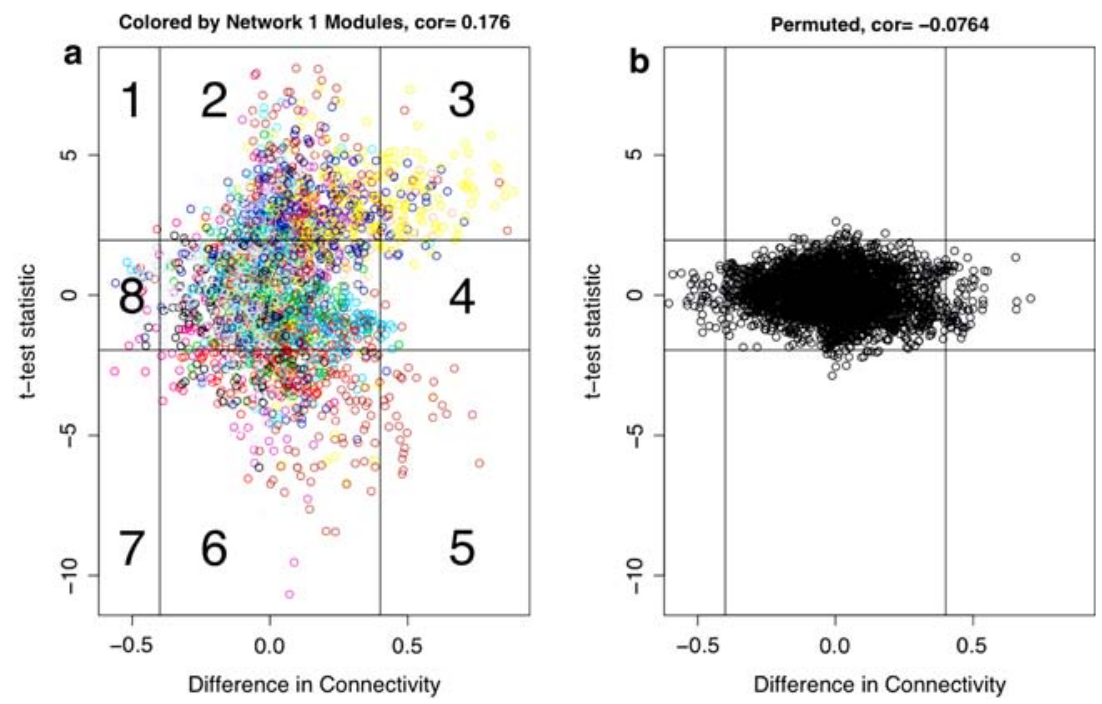

enriched for the extracellular region $(37.7 \%$ of genes $\left.p=1.8 \times 10^{-4}\right)$, extracellular space $(34.4 \%$ of genes $p=$ $\left.5.7 \times 10^{-4}\right)$, signaling $\left(36.1 \%\right.$ of genes $\left.p=5.4 \times 10^{-4}\right)$, cell adhesion $\left(16.4 \%\right.$ of genes $\left.p=7.7 \times 10^{-4}\right)$, and glycoproteins $\left(34.4 \%\right.$ of genes $\left.p=1.6 \times 10^{-3}\right)$. Furthermore, 12 terms for epidermal growth factor or its related proteins were recovered in the functional analysis. A few of the notable results are EGF-like $1\left(8.2 \%\right.$ of genes $\left.p=8.7 \times 10^{-4}\right)$, EGFlike $3\left(6.6 \%\right.$ of genes $\left.p=1.6 \times 10^{-3}\right)$, EGF-like $2(6.6 \%$ of genes $\left.p=6.0 \times 10^{-3}\right)$, EGF $(8.2 \%$ of genes $p=0.013)$, and EGF_CA (6.6\% of genes $p=0.015)$.

In summary, we find a group of rewired genes identified by differential connectivity in lean and obese mice. These genes are highly enriched for extracellular and cell-cell interactions and notably 12 epidermal growth factor (EGF) or EGF-related factors. An indirect validation of the differential network results is provided by a published article that reports that EGF plays a causal role in inducing obesity in ovariectomized mice (Kurachi et al. 1993).

Functional enrichment analysis of sector 5 genes

Sector 5 is analagous to sector 3 in that it contains genes with both extreme differences in connectivity and extreme $t$-statistic values. After Bonferroni correction, these genes are enriched for enzyme inhibitor activity $\left(p=2.93 \times 10^{-3}\right)$,

Table 4 Functional enrichment analysis of the results of the differential network analysis

\begin{tabular}{|c|c|c|c|c|}
\hline Category & Term & Gene count & $\%$ Genes & $p$ Value \\
\hline GOTERM_CC_ALL & Extracellular region & 23 & $37.7 \%$ & $1.8 \times 10^{-4}$ \\
\hline UP_SEQ_FEATURE & Signal peptide & 22 & $36.1 \%$ & $5.4 \times 10^{-4}$ \\
\hline GOTERM_BP_ALL & Cell adhesion & 10 & $16.4 \%$ & $7.7 \times 10^{-4}$ \\
\hline UP_SEQ_FEATURE & Domain:EGF-like 1 & 5 & $8.2 \%$ & $8.7 \times 10^{-4}$ \\
\hline UP_SEQ_FEATURE & Glycosylation site:N-linked (GlcNAc...) & 21 & $34.4 \%$ & 0.0012 \\
\hline UP_SEQ_FEATURE & Domain:EGF-like 3 & 4 & $6.6 \%$ & 0.0016 \\
\hline SP_PIR_KEYWORDS & Cell adhesion & 7 & $11.5 \%$ & 0.0017 \\
\hline SP_PIR_KEYWORDS & Collagen & 5 & $8.2 \%$ & 0.0018 \\
\hline UP_SEQ_FEATURE & Domain:EGF-like 2 & 4 & $6.6 \%$ & 0.0060 \\
\hline SMART_NAME & SM00181:EGF & 5 & $8.2 \%$ & 0.013 \\
\hline SMART_NAME & SM00179:EGF_CA & 4 & $6.6 \%$ & 0.015 \\
\hline SP_PIR_KEYWORDS & egf-like domain & 5 & $8.2 \%$ & 0.017 \\
\hline INTERPRO_NAME & IPR000742:EGF-like, type 3 & 5 & $8.2 \%$ & 0.018 \\
\hline INTERPRO_NAME & IPR006210:EGF & 5 & $8.2 \%$ & 0.021 \\
\hline
\end{tabular}

We studied 61 differentially expressed and differentially connected genes in sector 3. Note the enrichment for EGF-related themes. Additional details including corrected $p$ values can be found in Supplementary Material, Appendix C 
protease inhibitor activity $\left(p=6.00 \times 10^{-3}\right)$, endopeptidase activity $\left(p=6.00 \times 10^{-3}\right), \quad$ dephosphorylation $(p=$ 0.0122), protein amino acid dephosphorylation $(p=0.0122)$, and serine-type endopeptidase inhibitor activity ( $p=0.0417$ ) (Supplementary Table 6). Two genes were enriched for all significant categories: Itihl and Itih3. These two genes are located near a QTL marker for hyperinsulinemia (D14Mit52) identified in C57B1/6, 129S6/ SvEvTac, and $(\mathrm{B} 6 \times 129) \mathrm{F}_{2}$ intercross mice (Almind and Kahn 2004). Itih 3 was independently determined to be a gene candidate for obesity-related traits based on differential expression in murine hypothalamus (Bischof and Wevrick 2005). Two serine protease inhibitors, Serpina $3 n$ and Serpina10, were enriched for the categories of enzyme inhibitor, protease inhibitor, and endopeptidase inhibitor. In humans, Serpina10 is also known as Protein Z-dependent protease inhibitor (ZPI). This serpin inhibits activated coagulation factors $\mathrm{X}$ and XI; ZPI deficiencies have been found to be associated with venous thrombosis (Water et al. 2004). We note that obesity is a strong independent risk factor for venous thrombosis (Abdollahi et al. 2003; Goldhaber et al. 1997) and that accordingly PZI may be a link between obesity and increased risk of venous thrombotic events.

Results from functional enrichment analysis for all other sectors are described in Supplementary Material, Appendix C and Supplementary Tables 3, 4, 5, 7, and 8 (Supplementary Table 3: enrichment of biological pathways/sets for Blue module genes intersecting $\mathrm{B} \times \mathrm{H}$ and B $\times$ D data sets; Supplementary Table 4: enrichment of biological pathways/sets for sector 2 genes; Supplementary Table 5: enrichment of biological pathways/sets for all sector 3 genes; Supplementary Table 7: enrichment of biological pathways/sets for sector 6 genes; Supplementary Table 8: enrichment of biological pathways/sets for sector 8 genes).

\section{Discussion}

Integrating weighted gene coexpression network analysis with genotype data holds great promise for elucidating the molecular and genetic basis of complex diseases. Since WGCNA focuses on coexpression modules (as opposed to individual gene expressions), it will be useful only if traitrelated modules can be detected in the gene expression data. In our mouse genetics application, we provide evidence for a body weight-related module that can be found in two $\mathrm{F}_{2}$ mouse crosses.

We show that several modules identified in the $\mathrm{F}_{2} \mathrm{~B} \times \mathrm{H}$ mouse intercross are roughly preserved in an independent $\mathrm{B} \times \mathrm{D}$ mouse cross. In particular, the weight-related module found in the $\mathrm{F}_{2}$ mouse intercross is recovered in the second mouse cross. Highly connected hub genes within this module are found to have high correlation with weight (GSweight). We also find that module-based measures tend to be stable and robust across independent data sets. This is even more striking given the difference between the $\mathrm{B} \times \mathrm{H}$ and $\mathrm{B} \times \mathrm{D}$ mouse populations. Hub gene status is also roughly preserved, validating the importance and robustness of intramodular connectivity. These validation successes provide evidence for the utility and robustness of network-based methods.

Central to WGCNA is the concept of intramodular connectivity, which can be considered a measure of module membership. In coexpression networks, intramodular hub genes can be considered the most central genes inside the module. Because the expression profiles of intramodular hub genes inside an interesting module are highly correlated, they are statistically equivalent. This does not imply that such genes have the same functional significance. Gene ontology may reveal that they differ in terms of biological plausibility or clinical utility. In many applications, the list of module hub genes may be further prioritized based on (1) biological plausibility based on external gene (ontology) information, (2) availability of protein biomarkers for further validation, (3) availability of suitable mouse models for further validation, and/or (4) druggability, i.e., the opportunity for therapeutic intervention.

We demonstrate that both single-network and differential network analyses may be useful for finding body weight-related genes. Single-network analysis describes the module structure and topological properties of a single data set. In single-network analysis, all samples, irrespective of their clinical trait, are used for network and module construction. In contrast, differential network analysis compares two different networks. Differential network analysis aims to identify genes that are both differentially expressed and differentially connected. Since module genes tend to be highly connected in coexpression networks, screening for differentially connected genes is related to studying the preservation of modules between the two networks. We have shown that genes that are differentially connected may or may not be differentially expressed. Changes in connectivity may correspond to large-scale "rewiring" in response to environmental changes and physiologic perturbations (Luscombe et al. 2004).

The availability of genetic markers greatly enhances the kind of questions that can be addressed by WGCNA. Genetic marker data provide valuable information for prioritizing gene expressions inside a module. The resulting systems genetics gene-screening strategy goes beyond drafting lists of differentially expressed genes or finding chromosomal locations that seem to cosegregate with a trait. 
Acknowledgments The authors acknowledge the support from Program Project Grant 1U19AI063603-01 and from NIH/NIDDKDDK072206. They are grateful for discussions with collaborators Jun Dong, Dan Geschwind, Peter Langfelder, Ai Li, Wen Lin, Paul Mischel, Stan Nelson, Roel Ophoff, Anja Presson, Christiaan Saris, Lin Wang, and Wei Zhao.

\section{References}

Abdollahi M, Cushman M, Rosendaal FR (2003) Obesity: risk of venous thrombosis and the interaction with coagulation factor levels and oral contraceptive use. Thromb Haemost 89:493-498

Almind K, Kahn CR (2004) Genetic determinants of energy expenditure and insulin resistance in diet-induced obesity in mice. Diabetes 53:3274-3285

Bischof JM, Wevrick R (2005) Genome-wide analysis of gene transcription in the hypothalamus. Physiol Genomics 22:191196

Bystrykh L, Weersing E, Dontje B, Sutton S, Pletcher MT, et al. (2005) Uncovering regulatory pathways that affect hematopoietic stem cell function using 'genetical genomics'. Nat Genet 37:225-232

Chen J, Lipska BK, Halim N, Ma QD, Matsumoto M, et al. (2004) Functional analysis of genetic variation in catechol-O-methyltransferase (COMT): effects on mRNA, protein, and enzyme activity in postmortem human brain. Am J Hum Genet 75:807821

Chesler EJ, Lu L, Shou S, Qu Y, Gu J, et al. (2005) Complex trait analysis of gene expression uncovers polygenic and pleiotropic networks that modulate nervous system function. Nat Genet 37:233-242

Dennis G Jr, Sherman BT, Hosack DA, Yang J, Gao W, et al. (2003) DAVID: Database for Annotation, Visualization, and Integrated Discovery. Genome Biol 4:P3

Drake TA, Schadt EE, Lusis AJ (2006) Integrating genetic and gene expression data: application to cardiovascular and metabolic traits in mice. Mamm Genome 17:466-479

Eppig JT, Bult CJ, Kadin JA, Richardson JE, Blake JA, et al. (2005) The Mouse Genome Database (MGD): from genes to mice-a community resource for mouse biology. Nucleic Acids Res 33:D471-D475

Ghazalpour A, Doss S, Sheth SS, Ingram-Drake LA, Schadt EE, et al. (2005) Genomic analysis of metabolic pathway gene expression in mice. Genome Biol 6:R59

Ghazalpour A, Doss S, Zhang B, Wang S, Plaisier C, et al. (2006) Integrating genetic and network analysis to characterize genes related to mouse weight. PLoS Genet 2:e130

Goldhaber SZ, Grodstein F, Stampfer MJ, Manson JE, Colditz GA, et al. (1997) A prospective study of risk factors for pulmonary embolism in women. JAMA 277:642-645

Horvath S, Zhang B, Carlson M, Lu KV, Zhu S, et al. (2006) Analysis of oncogenic signalling networks in glioblastoma identifies ASPM as a novel molecular target. Proc Natl Acid Sci 103(46):17402-17407

Hubner N, Wallace CA, Zimdahl H, Petretto E, Schulz H, et al. (2005) Integrated transcriptional profiling and linkage analysis for identification of genes underlying disease. Nat Genet 37:243253

Kadarmideen HN, von Rohr P, Janss LL (2006) From genetical genomics to systems genetics: potential applications in quantitative genomics and animal breeding. Mamm Genome 17:548564

Kaschina E, Stoll M, Sommerfeld M, Steckelings UM, Kreutz R, et al. (2004) Genetic kininogen deficiency contributes to aortic aneurysm formation but not to atherosclerosis. Physiol Genomics 19:41-49

Kurachi H, Adachi H, Ohtsuka S, Morishige K, Amemiya K, et al. (1993) Involvement of epidermal growth factor in inducing obesity in ovariectomized mice. Am J Physiol 265:E323-E331

Luscombe NM, Babu MM, Yu H, Snyder M, Teichmann SA, et al. (2004) Genomic analysis of regulatory network dynamics reveals large topological changes. Nature 431:308-312

Mahr S, Burmester GR, Hilke D, Gobel U, Grutzkau A, et al. (2006) Cis- and trans-acting gene regulation is associated with osteoarthritis. Am J Hum Genet 78:793-803

Nishimura DY, Swiderski RE, Searby CC, Berg EM, Ferguson AL, et al. (2005) Comparative genomics and gene expression analysis identifies BBS9, a new Bardet-Biedl syndrome gene. Am J Hum Genet 77:1021-1033

Nordstrom EA, Ryden M, Backlund EC, Dahlman I, Kaaman M, et al. (2005) A human-specific role of cell death-inducing DFFA (DNA fragmentation factor-alpha)-like effector A (CIDEA) in adipocyte lipolysis and obesity. Diabetes 54:1726-1734

Oldham MC, Horvath S, Geschwind DH (2006) Conservation and evolution of gene coexpression networks in human and chimpanzee brains. Proc Natl Acad Sci U S A 103:17973-17978

Raikwar NS, Cho WK, Bowen RF, Deeg MA (2006) Glycosylphosphatidylinositol-specific phospholipase D influences triglyceride-rich lipoprotein metabolism. Am J Physiol Endocrinol Metab 290:E463-E470

Reiner AP, Carlson CS, Rieder MJ, Siscovick DS, Liu K, et al. (2007) Coagulation factor VII gene haplotypes, obesity-related traits, and cardiovascular risk in young women. $\mathbf{J}$ Thromb Haemost 5:42-49

Schadt EE, Lum PY (2006) Thematic review series: systems biology approaches to metabolic and cardiovascular disorders. Reverse engineering gene networks to identify key drivers of complex disease phenotypes. J Lipid Res 47:2601-2613

Schadt EE, Monks SA, Drake TA, Lusis AJ, Che N, et al. (2003) Genetics of gene expression surveyed in maize, mouse and man. Nature 422:297-302

Viles-Gonzalez JF, Fuster V, Badimon JJ (2006) Links between inflammation and thrombogenicity in atherosclerosis. Curr Mol Med 6:489-499

Wang S, Yehya N, Schadt EE, Wang H, Drake TA, et al. (2006) Genetic and genomic analysis of a fat mass trait with complex inheritance reveals marked sex specificity. PLoS Genet 2:e15

Water N, Tan T, Ashton F, O'Grady A, Day T, et al. (2004) Mutations within the protein $\mathrm{Z}$-dependent protease inhibitor gene are associated with venous thromboembolic disease: a new form of thrombophilia. Br J Haemotol 127:190-194

Zhang B, Horvath S (2005) A general framework for weighted gene co-expression network analysis. Stat Appl Genet Mol Biol 4(1) Article 17 